\title{
SYMMETRIC COUPLING OF FINITE ELEMENTS AND BOUNDARY ELEMENTS FOR A PARABOLIC-ELLIPTIC INTERFACE PROBLEM
}

\author{
$\mathrm{BY}$ \\ M. COSTABEL (Technische Hochschule Darmstadt, Federal Republic of Germany) \\ V. J. ERVIN (Clemson University, Clemson, South Carolina) \\ AND \\ E. P. STEPHAN (Georgia Institute of Technology, Atlanta, Georgia)
}

0. Introduction. In [9], MacCamy and Suri study approximation methods for an interface problem in which Laplace's equation in one domain in $\mathbb{R}^{2}$ is coupled with the heat equation in another domain. This problem describes time-dependent eddy currents in two-dimensional electrodynamics. MacCamy and Suri use a finite element discretization for the spatial part of the heat equation and a boundary element method for the Laplace equation. They apply the standard coupling method for finite elements and boundary elements as analyzed by Johnson and Nedelec [7]. For smooth boundaries, they prove convergence of their semidiscretized Galerkin scheme which leads to a system of ordinary differential equations in time. An analysis of a fully discretized version of their coupling scheme is not available and will be difficult, because the stiffness matrix in this coupling method is neither symmetric nor positive definite.

In $[2,3,4,5,6]$ a symmetric method for the coupling of finite element and boundary element methods was developed and applied to various interface problems. In this paper, we show that this coupling method can be successfully applied also to the parabolic-elliptic interface problem studied by MacCamy and Suri. We follow MacCamy and Suri in our convergence proof for the semidiscrete Galerkin scheme. After discretization in space, we obtain an initial value problem for a system of ordinary differential equations whose stiffness matrix is positive definite. This allows us to apply a Crank-Nicolson method for its solution. We prove convergence for this fully discretized Galerkin scheme. Several numerical examples confirm the feasibility of our method and the theoretical convergence rates.

1. The coupling procedure and semidiscrete Galerkin scheme. Let $\Omega_{1}$ be a bounded, simply connected domain in $\mathbb{R}^{2}$ with Lipschitz boundary $\Gamma$ and complement $\Omega_{2}=$ $\mathbb{R}^{2} \backslash \bar{\Omega}$. We require without loss of generality for the analytic capacity $\operatorname{cap}(\Gamma)<1$ 
which can always be obtained by scaling. We consider the following parabolic-elliptic interface problem: For $T>0$ and given functions $q$ in $\Omega_{1}, \tilde{f}$ in $\Omega_{1} \times(0, T)$ and $\tilde{v}_{0}, \tilde{\psi}_{0}$ on $\Gamma \times(0, T)$ find $v_{1}, v_{2}, \tilde{A}$ such that

$$
\begin{gathered}
\frac{\partial v_{1}}{\partial t}=\Delta v_{1}+\tilde{f} \text { in } \Omega_{1} \times(0, T), \quad \Delta v_{2}=0 \quad \text { in } \Omega_{2} \times(0, T) \\
v_{1}-v_{2}=\tilde{v}_{0}, \quad \frac{\partial v_{1}}{\partial n}-\frac{\partial v_{2}}{\partial n}=\tilde{\psi}_{0} \quad \text { on } \Gamma \times(0, T) \\
\left.v_{1}\right|_{t=0}=q \text { in } \Omega_{1}, \quad v_{2}=\tilde{A}(t) \log |x|+O\left(\frac{1}{|x|}\right) \quad \text { as }|x| \rightarrow \infty .
\end{gathered}
$$

In (1.2) $\partial v / \partial n$ means the derivative of $v$ with respect to the normal on $\Gamma$ pointing from $\Omega_{1}$ into $\Omega_{2}$.

Now, we choose a constant $\lambda>0$ and introduce

$$
u_{1}:=e^{-\lambda t} v_{1} \quad \text { in } \Omega_{1} \times(0, T), \quad u_{2}:=e^{-\lambda t} v_{2} \quad \text { in } \Omega_{2} \times(0, T) .
$$

Then with

$$
f:=e^{-\lambda t} \tilde{f}, \quad v_{0}:=e^{-\lambda t} \tilde{v}_{0}, \quad \psi_{0}:=e^{-\lambda t} \tilde{\psi}_{0}, \quad A:=e^{-\lambda t} \tilde{A}
$$

we obtain the interface problem

$$
\begin{array}{ccc}
\frac{\partial u_{1}}{\partial t}=\Delta u_{1}-\lambda u_{1}+f & \text { in } \Omega_{1} \times(0, T), \quad \Delta u_{2}=0 & \text { in } \Omega_{2} \times(0, T) \\
u_{1}-u_{2}=v_{0}, & \frac{\partial u_{1}}{\partial n}-\frac{\partial u_{2}}{\partial n}=\psi_{0} & \text { on } \Gamma \times(0, T) \\
\left.u_{1}\right|_{t=0}=q \quad \text { in } \Omega_{1}, & u_{2}=A(t) \log |x|+O\left(\frac{1}{|x|}\right) & (|x| \rightarrow \infty) .
\end{array}
$$

First we give a variational formulation for the "interior" problem in $\Omega_{1}$. Application of Green's first formula to $(1.1)^{\prime}$ yields for all $u, w \in C^{\infty}\left([0, T] ; C^{\infty}\left(\bar{\Omega}_{1}\right)\right)$ with $\dot{u}:=\partial u / \partial t$

$$
\int_{\Omega_{1}} w \dot{u} d x+\int_{\Omega_{1}} \nabla w \cdot \nabla u d x+\lambda \int_{\Omega_{1}} u w d x-\int_{\Gamma} w \frac{\partial u}{\partial n} d s=\int_{\Omega_{1}} w f d x .
$$

Next we take the second Green's formula in $\Omega_{2}$

$$
\int_{\Omega_{2}}(w \Delta u-u \Delta w) d x=\int_{\Gamma}\left(u \frac{\partial w}{\partial n}-w \frac{\partial u}{\partial n}\right) d s .
$$

Insertion of the fundamental solution $G(x, y)=-\frac{1}{2 \pi} \ln (x-y)$ of $-\Delta u=0$ gives the representation formula for $x \in \Omega_{2}$

$$
u_{2}(x)=\int_{\Gamma}\left\{\frac{\partial}{\partial n_{y}} G(x, y) v(y)-G(x, y) \phi(y)\right\} d s_{y}
$$

where

$$
v=\left.u_{2}\right|_{\Gamma}, \quad \phi=\left.\frac{\partial u_{2}}{\partial n}\right|_{\Gamma} .
$$


Taking Cauchy data in (1.6) one finds the relations on $\Gamma$

$$
\left[\begin{array}{l}
v \\
\phi
\end{array}\right]=\mathscr{C}\left[\begin{array}{l}
v \\
\phi
\end{array}\right]
$$

where the Calderón projector

$$
\mathscr{C}=\left[\begin{array}{ll}
\frac{1}{2}+\Lambda & -V \\
-D & \frac{1}{2}-\Lambda^{\prime}
\end{array}\right]
$$

is defined via the boundary integral operators:

$$
\begin{gathered}
V v(x)=\int_{\Gamma} G(x, y) v(y) d s_{y}, \quad \Lambda v(x)=\int_{\Gamma} \frac{\partial}{\partial n_{y}} G(x, y) v(y) d s_{y} \\
D v(x)=-\frac{\partial}{\partial n_{x}} \int_{\Gamma} \frac{\partial}{\partial n_{y}} G(x, y) v(y) d s_{y}, \quad \Lambda^{\prime} v(x)=\int_{\Gamma} \frac{\partial}{\partial n_{x}} G(x, y) v(y) d s_{y} .
\end{gathered}
$$

Following Costabel [2] one obtains a symmetric coupling method if one adds to (1.4) the weak form of the system (1.7) on the boundary $\Gamma$. Thus we define the bilinear form

$$
\begin{aligned}
a(u, \phi ; w, \psi)= & \int_{\Omega_{1}}(\nabla u \cdot \nabla w+\lambda u w) d x \\
& +\int_{\Gamma}\left\{w D u-w\left(\frac{1}{2}-\Lambda^{\prime}\right) \phi-\psi u+\psi\left(\frac{1}{2}+\Lambda\right) u-\psi V \phi\right\} d s
\end{aligned}
$$

for $u, w \in L^{2}\left(0, T ; H^{1}\left(\Omega_{1}\right) ; \phi, \psi \in L^{2}\left(0, T ; H^{-1 / 2}(\Gamma)\right)\right.$. For the definition of the Sobolev spaces $L^{2}\left(0, T ; H^{1}\left(\Omega_{1}\right)\right), L^{2}\left(0, T ; H^{-1 / 2}(\Gamma)\right)$ etc., (see [8]). With the given data $v_{0} \in L^{2}\left(0, T ; H^{1 / 2}(\Gamma)\right), \psi_{0} \in L^{2}\left(0, T ; H^{-1 / 2}(\Gamma)\right), f \in$ $L^{2}\left(0, T ; H^{1}\left(\Omega_{1}\right)^{\prime}\right)$ we have the linear form

$$
\ell(w, \psi)=\int_{\Gamma}\left\{w \psi_{0}+w D v_{0}-\psi v_{0}+\psi\left(\frac{1}{2}+\Lambda\right) v_{0}\right\} d s+\int_{\Omega_{1}} w f d x
$$

We consider the variational problem: Given $f \in L^{2}\left(0, T ; H^{1}\left(\Omega_{1}\right)^{\prime}\right), v_{0} \in$ $L^{2}\left(0, T ; H^{1 / 2}(\Gamma)\right), \quad \psi_{0} \in L^{2}\left(0, T ; H^{-1 / 2}(\Gamma)\right)$ find $u \in Q_{T}$ and $\phi \in B_{T}:=$ $L^{2}\left(0, T ; H^{-1 / 2}(\Gamma)\right)$ satisfying

$$
\int_{\Omega_{1}} w \dot{u} d x+a(u, \phi ; w, \psi)=\ell(w, \psi)
$$

for all $w \in L^{2}\left(0, T ; H^{1}\left(\Omega_{1}\right)\right), \psi \in L^{2}\left(0, T ; H^{-1 / 2}(\Gamma)\right)$ where

$$
Q_{T}=\left\{u \in L^{2}\left(0, T ; H^{1}\left(\Omega_{1}\right)\right): \dot{u} \in L^{2}\left(0, T ;\left(H^{1}\left(\Omega_{1}\right)\right)^{\prime}\right),\left.u\right|_{t=0}=q\right\} .
$$

Note that $u \in H_{T}:=L^{2}\left(0, T ; H^{1}\left(\Omega_{1}\right)\right)$ and $\dot{u} \in H_{T}^{\prime}:=L^{2}\left(0, T ;\left(H^{1}\left(\Omega_{1}\right)\right)^{\prime}\right)$ implies $u \in C\left([0, T] ; L^{2}\left(\Omega_{1}\right)\right)$ so that $\left.u\right|_{t=0}=q$ is well-defined.

Let $\left\{H_{h}^{1}\right\}$ and $\left\{H_{h}^{-1 / 2}\right\}$ be families of finite dimensional subspaces of $H^{1}\left(\Omega_{1}\right)$ and $H^{-1 / 2}(\Gamma)$, respectively, and let $\left\{H_{T}^{h}\right\}$ and $\left\{B_{T}^{h}\right\}$ be subspaces of $L^{2}\left(0, T ; H_{h}^{1}\right)$ and $L^{2}\left(0, T ; H_{h}^{-1 / 2}\right)$, respectively. 
The corresponding Galerkin scheme is: Find $\left(u_{h}, \phi_{h}\right) \in H_{T}^{h} \times B_{T}^{h}$ such that for all $\left(w_{h}, \psi_{h}\right) \in H_{h}^{1} \times H_{h}^{-1 / 2}$

$$
\int_{\Omega_{1}} w_{h} \frac{\partial u_{h}}{\partial t}+a\left(u_{h}, \phi_{h} ; w_{h}, \psi_{h}\right)=\ell\left(w_{h}, \psi_{h}\right) \quad \text { for } t>0
$$

and

$$
\left.u_{h}\right|_{t=0}=P_{h} q, \quad \text { i.e., } \int_{\Omega_{1}} u_{h}(0, x) w_{h}(x) d x=\int_{\Omega_{1}} q(x) w_{h}(x) d x .
$$

We follow closely the presentation in [9] and use the convergence proof of the semidiscrete Galerkin scheme (1.14) to obtain an existence proof for the continuous problem (1.12). Thus we need two assumptions concerning the spaces $H_{h}^{1}, H_{h}^{-1 / 2}, H_{T}^{h}$, and $B_{T}^{h}$ :

(A.1) Define the $L^{2}$-projection $P_{h}: H^{1}\left(\Omega_{1}\right) \rightarrow H_{h}^{1}$ by

$$
\int_{\Omega_{1}}\left(P_{h} v\right) w_{h} d x=\int_{\Omega_{1}} v w_{h} d x \quad \text { for all } w_{h} \in H_{h}^{1} .
$$

Then there is a constant $\tilde{\gamma}>0$, independent of $h$, such that

$$
\sup _{v \in H^{1}\left(\Omega_{1}\right)} \frac{\left\|P_{h} v\right\|_{1}}{\|v\|_{1}} \leq \tilde{\gamma}
$$

where $\|\cdot\|_{1}$ denotes the norm of $H^{1}\left(\Omega_{1}\right)$.

(A.2) Approximation property in $Q_{T} \times B_{T}$ : For any $\varepsilon>0$ there is an $h_{\varepsilon}$ such that for any $h \leq h_{\varepsilon}$ and $(u, \phi) \in Q_{T} \times B_{T}$ there is a $\left(u_{h}, \phi_{h}\right) \in H_{T}^{h} \times B_{T}^{h}$ such that

$$
\left\|u-u_{h}\right\|_{H_{T}}+\left\|\dot{u}-\dot{u}_{h}\right\|_{H_{T}^{\prime}}+\left\|\phi-\phi_{h}\right\|_{B_{T}} \leq \varepsilon
$$

where

$$
\|w\|_{H_{T}}:=\|w\|_{L^{2}\left(0, T ; H^{\prime}\left(\Omega_{1}\right)\right)}, \quad\|w\|_{H_{T}^{\prime}}:=\|w\|_{L^{2}\left(0, T:\left(H^{\prime}\left(\Omega_{1}\right)\right)^{\prime}\right)}
$$

and

$$
\|w\|_{B_{T}}:=\|w\|_{L^{2}\left(0, T: H^{-1 / 2}(\Gamma)\right)} .
$$

Theorem 1. Suppose (A.1), (A.2) hold. Then there exists a unique solution $(u, \phi) \in$ $Q_{T} \times B_{T}$ of (1.12) and it defines a solution of $(1.1)^{\prime}-(1.3)^{\prime}$ where $u_{1}=u$ and $u_{2}$ is given by the representation formula (1.6) with $v=\left.u\right|_{\Gamma}-v_{0}$. Moreover there is a constant $C$, independent of $T$, such that

$$
\|u\|_{Q_{T}}+\|\phi\|_{B_{T}} \leq C\left\{\left\|v_{0}\right\|_{B_{T}^{\prime}}+\left\|\psi_{0}\right\|_{B_{7}}+\|q\|_{L^{2}\left(\Omega_{1}\right)}+\|f\|_{H_{T}^{\prime}}\right\}
$$

where

$$
\|u\|_{Q_{T}}:=\|u\|_{H_{T}}+\|\dot{u}\|_{H_{T}^{\prime}}, \quad\left\|v_{0}\right\|_{B_{T}^{\prime}}:=\left\|v_{0}\right\|_{L^{2}\left(0, T: H^{1 / 2}(\Gamma)\right)^{\prime}}
$$

Proof. The proof is given in several steps: First we observe that the bilinear form $a(\cdot, \cdot)$ in $(1.10)$ satisfies the Babuška-Brezzi condition on $H^{1}\left(\Omega_{1}\right) \times H^{-1 / 2}(\Gamma)$ for 
fixed $t \in(0, T)$. Namely if we take $w=u$ and $\psi=-\phi$ in (1.10), we obtain with a uniform constant $\gamma$ (compare [5])

$$
a(u, \phi ; u,-\phi) \geq \gamma\left(\|u\|_{H^{1}\left(\Omega_{1}\right)}^{2}+\|\phi\|_{H^{-1 / 2}(\Gamma)}^{2}\right) .
$$

Next, to obtain estimates on $(u, \phi)$ for variable time $t$ we need the following two lemmas. So we interrupt the proof of Theorem 1.

Lemma 1. The Galerkin equations (1.14) have unique solutions for each $h$.

Proof. If we choose bases for $H_{T}^{h}$ and $B_{T}^{h}$ then (1.14) will be equivalent to

$$
\begin{gathered}
\mathscr{M} \dot{u}_{h}+(\mathscr{R}+\lambda \mathscr{M}) U_{h}+\mathscr{D} U_{h}+\mathscr{B} \Phi_{h}=F ; \quad U_{h}(0)=0 \\
S \Phi_{h}-\mathscr{B}^{T} U_{h}=G .
\end{gathered}
$$

Here $U_{h}$ and $F$ have values in $\mathbb{R}^{M}$ and $\Phi_{h}$ and $G$ have values in $\mathbb{R}^{N}$ for some $M$ and $N . \mathscr{M}, \mathscr{K}$, and $\mathscr{D}$ are $M \times M$ matrices, $\mathscr{B}$ is an $M \times N$ matrix, and $S$ is an $N \times N$ matrix. The mass matrix $\mathscr{M}$ is positive definite. Furthermore, $\mathscr{K}$ and $\mathscr{D}$ are positive semidefinite and $S$ is positive definite, since

$$
\begin{gathered}
\int_{\Omega_{1}} \nabla u \cdot \nabla u d x \geq 0, \quad \int_{\Omega_{1}}(\nabla u \cdot \nabla u+\lambda u u) d x \geq \lambda\|u\|_{H^{1}\left(\Omega_{1}\right)}^{2} \quad \text { for all } u \in H^{1}\left(\Omega_{1}\right) \\
\int_{\Gamma} u D u d s \geq 0 \quad \text { for all } u \in H^{1 / 2}(\Gamma)
\end{gathered}
$$

and there exists a constant $\lambda_{2}>0$ such that

$$
\int_{\Gamma} \phi V \phi d s \geq \lambda_{2}\|\phi\|_{H^{-1 / 2}(\Gamma)}^{2} \quad \text { for all } \phi \in H^{-1 / 2}(\Gamma) .
$$

(Note for $(1.21)$ to hold we need the condition $\operatorname{cap}(\Gamma)<1$.) Therefore, one can solve (1.18) for $\Phi_{h}$ in terms of $U_{h}$ and substitute into (1.17) to obtain a differential equation for $U_{h}$. Since $\mathscr{C}$ is nonsingular, this equation has a unique solution.

Next we show the solutions of the Galerkin scheme (1.14) are stable (with respect to the data).

LEMma 2. There exists a constant $C>0$, independent of $h$, such that the solution of (1.14) satisfies

$$
\left\|u_{h}\right\|_{Q_{T}}+\left\|\phi_{h}\right\|_{B_{T}} \leq C\left(\left\|v_{0}\right\|_{B_{T}^{\prime}}+\left\|\phi_{0}\right\|_{B_{T}}+\|q\|_{L^{2}\left(\Omega_{1}\right)}+\|f\|_{H_{T}^{\prime}}\right)
$$

and

$$
\left\|u_{h}\right\|_{L^{\infty}\left(0, T: L^{2}\left(\Omega_{1}\right)\right)} \leq C\left[\left\|v_{0}\right\|_{B_{T}^{\prime}}+\left\|\phi_{0}\right\|_{B_{T}}+\|q\|_{L^{2}\left(\Omega_{1}\right)}+\|f\|_{H_{T}^{\prime}}\right] .
$$

Proof. We consider the Galerkin equations (1.14) for fixed $t$ and insert $w_{h}=u_{h}$, $\psi_{h}=-\phi_{h}$ obtaining

$$
\frac{1}{2} \frac{d}{d t}\left\|u_{h}\right\|_{L^{2}\left(\Omega_{1}\right)}^{2}+a\left(u_{h}, \phi_{h} ; u_{h},-\phi_{h}\right)=\ell\left(u_{h},-\phi_{h}\right) .
$$


Therefore the coerciveness inequality (1.16) and the continuity of $a(\cdot, \cdot)$ give with constants $C, \varepsilon>0$

$$
\begin{aligned}
\frac{d}{d t} \| & u_{h}\left\|_{L^{2}\left(\Omega_{1}\right)}^{2}+\right\| u_{h}\left\|_{H^{1}\left(\Omega_{1}\right)}^{2}+\right\| \phi_{h} \|_{H^{-1 / 2}(\Gamma)}^{2} \\
& \leq C\left[\left\|u_{h}\right\|_{H^{1}\left(\Omega_{1}\right)}+\left\|\phi_{h}\right\|_{H^{-1 / 2}(\Gamma)}\right]\left(\left\|v_{0}\right\|_{H^{1 / 2}(\Gamma)}+\left\|\phi_{0}\right\|_{H^{-1 / 2}(\Gamma)}+\|f\|_{H^{1}\left(\Omega_{1}\right)^{\prime}}\right) \\
& \leq C\left\{\varepsilon\left(\left\|u_{h}\right\|_{H^{1}\left(\Omega_{1}\right)}^{2}+\left\|\phi_{h}\right\|_{H^{-1 / 2}(\Gamma)}^{2}\right)+\frac{1}{\varepsilon}\left(\left\|v_{0}\right\|_{H^{1 / 2}(\Gamma)}^{2}+\left\|\phi_{0}\right\|_{H^{-1 / 2}(\Gamma)}^{2}+\|f\|_{H^{1}\left(\Omega_{1}\right)^{\prime}}^{2}\right)\right\} .
\end{aligned}
$$

Hence for fixed $t$ we have

$$
\frac{d}{d t}\left\|u_{h}\right\|_{L^{2}\left(\Omega_{1}\right)}^{2} \leq C\left(\left\|v_{0}\right\|_{H^{1 / 2}(\Gamma)}^{2}+\left\|\phi_{0}\right\|_{H^{-1 / 2}(\Gamma)}^{2}+\|f\|_{H^{\prime}\left(\Omega_{1}\right)^{\prime}}^{2}\right) .
$$

Therefore integration with respect to $t$ from 0 to $t$ yields

$$
\left\|u_{h}(t)\right\|_{L^{2}\left(\Omega_{1}\right)}^{2} \leq C\left(\left\|v_{0}\right\|_{B_{T}^{\prime}}^{2}+\left\|\phi_{0}\right\|_{B_{T}}^{2}+\|f\|_{H_{T}^{\prime}}^{2}\right)+\|q\|_{L^{2}\left(\Omega_{1}\right)}^{2} .
$$

Hence

$$
\left\|u_{h}\right\|_{L^{\infty}\left(0, T: L^{2}\left(\Omega_{1}\right)\right)} \leq C\left[\left\|v_{0}\right\|_{B_{T}^{\prime}}+\left\|\phi_{0}\right\|_{B_{T}}+\|q\|_{L^{2}\left(\Omega_{1}\right)}+\|f\|_{H_{T}^{\prime}}\right] .
$$

On the other hand if we integrate in (1.24) over $t$ from 0 to $T$ we obtain

$$
\left\|u_{h}\right\|_{H_{T}}^{2}+\left\|\phi_{h}\right\|_{B_{T}}^{2} \leq C\left(\left\|v_{0}\right\|_{B_{T}^{\prime}}^{2}+\left\|\phi_{0}\right\|_{B_{T}}^{2}+\|q\|_{L^{2}\left(\Omega_{1}\right)}^{2}+\|f\|_{H_{T}^{\prime}}^{2}\right. \text {. }
$$

Now, let $P_{h}$ be the $L^{2}$ projection of (A.1). We have

$$
\left\|\dot{u}_{h}\right\|_{\left(H^{\prime}\left(\Omega_{1}\right)\right)^{\prime}}=\sup _{v \in H^{1}\left(\Omega_{1}\right)} \frac{\int_{\Omega_{1}} \dot{u}_{h} v d x}{\|v\|_{1}}=\sup _{v \in H^{\prime}\left(\Omega_{1}\right)} \frac{\int_{\Omega_{1}} \dot{u}_{h} P_{h} v d x}{\|v\|_{1}} .
$$

Now we use (1.14) with $w_{h}=P_{h} v$ and $\psi_{h}=0$ to estimate the right hand side of this equality.

$$
\begin{aligned}
&\left\|\dot{u}_{h}\right\|_{\left(H^{1}\left(\Omega_{1}\right)\right)^{\prime}=} \sup _{v \in H^{1}\left(\Omega_{1}\right)} \frac{1}{\|v\|_{1}}\left\{\int_{\Gamma}\left(D v_{0}+\psi_{0}\right) P_{h} v d s+\int_{\Omega_{1}} f P_{h} v d x-a\left(u_{h}, \phi_{h} ; P_{h} v, 0\right)\right\} \\
&=\sup _{v \in H^{1}\left(\Omega_{1}\right)} \frac{1}{\|v\|_{1}}\left\{\int_{\Gamma}\left(D v_{0}+\psi_{0}\right) P_{h} v d s-\int_{\Omega_{1}}\left(\nabla u_{h} \cdot \nabla\left(P_{h} v\right)+\lambda u_{h} P_{h} v\right) d x\right. \\
&\left.\quad+\int_{\Omega_{1}} f P_{h} v d x+\int_{\Gamma}\left(P_{h} v\right)\left(\left(\frac{1}{2}-\Lambda^{\prime}\right) \phi_{h}-D u_{h}\right) d s\right\} \\
& \leq C\left\{\left\|v_{0}\right\|_{H^{1 / 2}(\Gamma)}+\left\|\psi_{0}\right\|_{H^{-1 / 2}(\Gamma)}\right. \\
&\left.\quad+\|f\|_{H^{1}\left(\Omega_{1}\right)^{\prime}}+\left\|u_{h}\right\|_{H^{\prime}\left(\Omega_{1}\right)}+\left\|\phi_{h}\right\|_{H^{-1 / 2}(\Gamma)}\right\} \sup \frac{\left\|P_{h} v\right\|_{1}}{\|v\|_{1}} .
\end{aligned}
$$

Here we have used that by the trace lemma

$$
\left\|\left.\left(P_{h} v\right)\right|_{\Gamma}\right\|_{H^{1 / 2}(\Gamma)} \leq C\left\|P_{h} v\right\|_{H^{\prime}\left(\Omega_{1}\right)} .
$$


Now, taking squares on both sides of (1.26) and integrating over $t$ from 0 to $T$ using (A.1) and (1.25) yields the estimate

$$
\left\|\dot{u}_{h}\right\|_{L^{2}\left(0, T ;\left(H^{\prime}\left(\Omega_{1}\right)\right)^{\prime}\right)} \leq C\left\{\left\|v_{0}\right\|_{B_{T}^{\prime}}+\left\|\phi_{0}\right\|_{B_{T}}+\|q\|_{L^{2}\left(\Omega_{1}\right)}+\|f\|_{H_{T}^{\prime}}\right\} .
$$

Hence combining (1.25) and (1.27) we obtain (1.22).

Now we are in the position to complete the proof of Theorem 1: We conclude from the estimate (1.22) of Lemma 2 that there exists a subsequence of the sequence $\left\{\left(u_{h}, \phi_{h}\right)\right\}$ of Galerkin solutions which converges weakly to $(u, \phi)$ with $\left\{\dot{u}_{h}\right\}$ converging weakly to $u_{t}$. The existence of the Galerkin solutions is guaranteed by Lemma 1. Then one shows from (A.2) that $u_{t}$ is the generalized derivative of $u$ and that $(u, \phi)$ satisfies $(1.12)$, i.e., $(u, \phi)$ is a variational solution of our coupling problem. The estimate (1.15) follows from (1.22) and lower semi-continuity.

The next theorem gives the convergence and quasioptimality of the Galerkin solution.

Theorem 2. Suppose (A.1), (A.2) hold. Then there exists a $C>0$ such that

(i) the Galerkin scheme (1.14) has a unique solution $\left(u_{h}, \phi_{h}\right) \in H_{T}^{h} \times B_{T}^{h}$;

(ii)

$$
\begin{aligned}
& \left\|u-u_{h}\right\|_{Q_{T}}+\left\|\phi-\phi_{h}\right\|_{B_{T}} \\
& \quad \leq C \inf \left\{\left\|u-w_{h}\right\|_{Q_{T}}+\left\|\phi-\psi_{h}\right\|_{B_{T}}: w_{h} \in H_{T}^{h}, \psi_{h} \in B_{T}^{h}\right\}
\end{aligned}
$$

where $C$ is independent of $u, \phi, h$ and $T$.

The proof of Theorem 2 is standard and we only outline the main steps. First let us introduce the following definition.

Definition 1. The Galerkin operator $G_{h}$ for (1.14) is the projection from $Q_{T} \times B_{T}$ into $H_{T}^{h} \times B_{T}^{h}$ defined by $G_{h}(u, \phi)=(\tilde{u}, \tilde{\phi})$ where $\tilde{u}(x, 0)$ interpolates $q$ and for any $\left(w_{h}, \psi_{h}\right) \in H_{h}^{1} \times H_{h}^{-1 / 2}$

$$
\int_{\Omega_{1}} w_{h} \dot{\tilde{u}} d x+a\left(\tilde{u}, \tilde{\phi} ; w_{h}, \psi_{h}\right)=\int_{\Omega_{1}} w_{h} \dot{u} d x+a\left(u, \phi ; w_{h}, \psi_{h}\right) .
$$

Proof of Theorem 2. First we observe that as a consequence of the estimate (1.22) the Galerkin operator $G_{h}$ is bounded in the operator norm independent of $h$. Furthermore, from Lemma 1 it follows that $G_{h}$ is well defined. From the stability estimate (1.22) one obtains the convergence of $G_{h}(u, \phi)$ to $(u, \phi)$ in a standard way: Let $\left(z_{h}, \chi_{h}\right)$ be an arbitrary element of $H_{T}^{h} \times B_{T}^{h}$ and set $\left(e_{1}, e_{2}\right):=G_{h}(u, \phi)-\left(z_{h}, \chi_{h}\right),\left(\varepsilon_{1}, \varepsilon_{2}\right):=(u, \phi)-\left(z_{h}, \chi_{h}\right)$. Then (1.29) yields

$$
\int_{\Omega_{1}} w_{h} \dot{e}_{1} d x+a\left(e_{1}, e_{2} ; w_{h}, \psi_{h}\right)=\int_{\Omega_{1}} w_{h} \dot{\varepsilon}_{1} d x+a\left(\varepsilon_{1}, \varepsilon_{2} ; w_{h}, \psi_{h}\right) .
$$

The right hand side of (1.30) can be written in the same form as that in (1.14). Therefore the estimate (1.22) can be applied and we obtain

Hence

$$
\left\|e_{1}\right\|_{Q_{T}}+\left\|e_{2}\right\|_{B_{T}} \leq C\left(\left\|\varepsilon_{1}\right\|_{Q_{T}}+\left\|\varepsilon_{2}\right\|_{B_{T}}\right) .
$$

$$
\left\|G_{h}(u, \phi)-(u, \phi)\right\|_{Q_{T} \times B_{T}} \leq C \inf \left\{\left\|(u, \phi)-\left(z_{h}, \chi_{h}\right)\right\|_{Q_{T} \times B_{T}}: z_{h} \in H_{T}^{h}, \chi_{h} \in B_{T}^{h}\right\}
$$

which is the desired estimate (1.28). 
Following [9] we discuss approximate subspaces for which assumptions (A.1), (A.2) are satisfied and show that quasioptimal convergence is obtained for such spaces.

In the following we assume $\Omega_{1}$ is polygonal and its boundary $\Gamma$ consists of straight line segments. We introduce regular grids $\Delta_{\Omega_{1}}$ and $\Delta_{\Gamma}$ on $\Omega_{1}$ and $\Gamma$ respectively with generic mesh spacings $h_{\Omega_{1}}$ and $h_{\Gamma}$.

Let $H_{h}^{1} \subset H^{1}\left(\Omega_{1}\right)$ be a space of piecewise polynomials of degree $\leq k-1$ on $\Delta_{\Omega_{1}}$ and $H_{h}^{-1 / 2} \subset H^{-1 / 2}(\Gamma)$ be a space of piecewise polynomials of degree $\leq \ell-1$ on $\Delta_{\Gamma}$. We take $h=\max \left\{h_{\Omega}, h_{\Gamma}\right\}$.

The trial functions $u_{h} \in H_{T}^{h}, \phi_{h} \in B_{T}^{h}$ in the Galerkin scheme (1.14) are timedependent whereas the test functions $w_{h} \in H_{h}^{1}, \psi_{h} \in H_{h}^{-1 / 2}$ do not depend on time. Therefore we set with $H_{h}^{1}$ and $H_{h}^{-1 / 2}$ as defined above

$$
\begin{gathered}
H_{T}^{h}:=\left\{v_{h} \in C^{1}\left([0, T] ; H_{h}^{1}\right), v_{h}=0 \text { at } t=0\right\} \\
B_{T}^{h}:=C^{0}\left([0, T] ; H_{h}^{-1 / 2}\right) .
\end{gathered}
$$

Now, with the above choices, the spaces $H_{T}^{h} \times B_{T}^{h}$ approximate the space $Q_{T} \times B_{T}$ in the sense of assumption (A.2).

For simplicity we consider only the case $k=2$ and $\ell=1$, i.e., piecewise linear, continuous finite elements in $\Omega_{1}$ and piecewise constant boundary elements on $\Gamma$. Let

$$
\begin{array}{r}
Z=\left\{(v, \psi), v \in L^{2}\left(0, T ; H^{2}\left(\Omega_{1}\right)\right), \dot{v} \in L^{2}\left(0, T ; H^{1}\left(\Omega_{1}\right)\right),\right. \\
\left.\left.v\right|_{t=0}=0, \psi \in L^{2}\left(0, T ; H^{1 / 2}(\Gamma)\right)\right\} .
\end{array}
$$

It is well known (see [1]) that for $(v, \psi) \in Z$

$$
\inf _{w_{h}^{\prime} \in H_{T}^{h}}\left\|v-w_{h}\right\|_{H_{T}}+\inf _{\chi_{h} \in B_{r}^{h}}\left\|\psi-\chi_{h}\right\|_{B_{r}} \leq C h
$$

where $C$ depends on $(v, \psi)$ but not on $h$. Moreover $v \in L^{2}\left(0, T ; H^{1}\left(\Omega_{1}\right)\right)$, $\dot{v} \in L^{2}\left(0, T ; H^{1}\left(\Omega_{1}\right)\right)$ can be approximated simultaneously by a function $w_{h} \in H_{T}^{h}$ and its derivative such that for almost all $t$ there holds

$$
\inf _{w_{h} \in H_{h}^{1}}\left\|v-w_{h}\right\|_{H^{\prime}\left(\Omega_{1}\right)} \leq C h\|v\|_{H^{2}\left(\Omega_{1}\right)}
$$

together with

$$
\left\|\dot{v}(t)-\dot{w}_{h}(t)\right\|_{L^{2}\left(\Omega_{1}\right)} \leq C h\|\dot{v}(t)\|_{H^{\prime}\left(\Omega_{1}\right)} .
$$

Integrating (1.34) in time and combining it with (1.33) we obtain for any $(v, \psi) \in Z$

$$
\inf _{w_{h}^{\prime} \in H_{T}^{h}}\left\{\left\|v-w_{h}\right\|_{H_{T}}+\left\|\dot{v}-\dot{w}_{h}\right\|_{H_{I}^{\prime}}\right\}+\inf _{\chi_{h} \in B_{l}^{h}}\left\|\psi-\chi_{h}\right\|_{B_{I}} \leq C h .
$$

Hence, since $Z$ is a dense subspace of $Q_{T} \times B_{T}$ we observe that for all $(v, \psi) \in$ $Q_{T} \times B_{T}$

$$
\inf \left\|(v, \psi)-\left(w_{h}, \chi_{h}\right)\right\|_{Q_{1} \times B_{l}} \rightarrow 0 \quad \text { as } h \rightarrow 0
$$


where the infinum is taken over all $\left(w_{h}, \chi_{h}\right) \in H_{T}^{h} \times B_{T}^{h}$. Therefore (A.2) holds for (1.31).

Furthermore (A.1) holds for $H_{T}^{h}$ in (1.31) if $H_{T}^{h}$ has an inverse property, i.e., there exists $0<C<\infty$ such that for all $v_{h} \in H_{T}^{h}$ and a.e. $t \in(0, T)$

$$
\left\|v_{h}\right\|_{H^{1}\left(\Omega_{1}\right)} \leq C h_{\Omega_{1}}^{-1}\left\|v_{h}\right\|_{L^{2}\left(\Omega_{1}\right)} \text {. }
$$

Finally, by combining (1.35) and (1.28) we obtain as a consequence of Theorem 2 the following convergence result. (Here, let $H_{h}^{1}$ consist of continuous piecewise linear finite elements and $H_{h}^{-1 / 2}$ of piecewise constant boundary elements.)

Corollary 1. Suppose the solution $(u, \phi)$ of $(1.12)$ belongs to the space $Z$ in (1.32) and suppose $H_{T}^{h}, B_{T}^{h}$ are given by (1.31) and the grid $\Delta_{\Omega_{1}}$ is regular. Then there holds for the solution $\left(u_{h}, \phi_{h}\right) \in H_{T}^{h} \times B_{T}^{h}$ of the semidiscrete Galerkin scheme (1.14)

$$
\begin{gathered}
\left\|u_{h}-u\right\|_{H_{T}}+\left\|\dot{u}_{h}-\dot{u}\right\|_{H_{T}^{\prime}}+\left\|\phi-\phi_{h}\right\|_{B_{T}} \leq C_{1} h, \\
\left\|u_{h}-u\right\|_{L^{\infty}\left(0, T: L^{2}\left(\Omega_{1}\right)\right)} \leq C_{2} h, \\
\left\|u_{h}-u\right\|_{L^{2}\left(0, T: L^{2}\left(\Omega_{1}\right)\right)} \leq C_{3} h^{2},
\end{gathered}
$$

where the constants $C_{1}, C_{2}, C_{3}$ depend on $(u, \phi)$ but do not depend on $h$.

Proof. For a regular grid $\Delta_{\Omega_{1}}$ the space $H_{T}^{h}$ as defined in (1.31) satisfies (1.36) where $H_{h}^{1}$ is the space of continuous, piecewise linear polynomials on $\Delta_{\Omega}$. Hence (A.1), (A.2) hold and therefore (1.37) is a direct consequence of (1.35) and (1.28). The estimate (1.38) follows by interpolation from (1.37).

2. A fully discrete coupling method. The semi-discretization reduces approximating the parabolic-elliptic interface problem $(1.1)^{\prime}-(1.3)^{\prime}$ to solving a (stiff) system of ordinary differential equations.

In general this cannot be done. Therefore, we must discretize the time variable to approximate the solution of the system of O.D.E.'s. One such method is the Crank-Nicolson method:

We seek to calculate sequences $\left\{U_{h}^{n}\right\} \subset H_{h}^{1},\left\{\phi_{h}^{n}\right\} \subset H_{h}^{-1 / 2}, n=0, \ldots, J$ where $J k=T$ and $U_{h}^{n} \approx u(n k), \phi_{h}^{n} \approx \phi(n k)$ and $U_{h}^{n}, \phi_{h}^{n}$ satisfy for all $w_{h} \in H_{h}^{1}$, $\psi_{h} \in H_{h}^{-1 / 2}$

$$
\begin{gathered}
\int_{\Omega_{1}} w_{h} \frac{U_{h}^{n+1}-U_{h}^{n}}{k} d x+a\left(\frac{U_{h}^{n+1}+U_{h}^{n}}{2}, \frac{\phi_{h}^{n+1}+\phi_{h}^{n}}{2}, w_{h}, \psi_{h}\right) \\
=\int_{\Gamma} \frac{g^{n+1}+g^{n}}{2} \psi_{h} d s+\int_{\Gamma} w_{h} \frac{f^{n+1}+f^{n}}{2} d s, \\
0 \leq n \leq J-1 \quad \text { with } U_{h}^{0}=P_{q}
\end{gathered}
$$

where $g^{n}(x):=\left(B v_{0}-v_{0}\right)(x, n k), f^{n}(x):=\left(\psi_{0}+D v_{0}\right)(x, n k)$ and $P$ is the $L^{2}$ projection. 
We note that to implement (4.2) we let $U_{h}^{n+1}=\sum_{i=1}^{m} c_{i}^{n+1} v_{i}(x)$ and set $w=v_{i}$ in (4.1), correspondingly we take $\phi_{h}^{n+1}=\sum_{i=1}^{\prime} d_{i}^{n+1} \chi_{i}(x)$ and set $\psi=\chi_{i}$ with basis functions $v_{i}, \chi_{i}$ of $H_{h}^{1}$ and $H_{h}^{-1 / 2}$, respectively. Thus at each time step we must solve

$$
\mathscr{M} \mathbf{c}^{n+1}+\frac{k}{2}\left[A \mathbf{c}^{n+1}+B \mathbf{d}^{n+1}\right]=\mathbf{b}^{n},
$$

with $A=\mathscr{K}+\lambda \mathscr{M}+\mathscr{D}-\mathscr{B}^{T}$ and $B=\mathscr{B}+S$ (compare (1.17), (1.18)) where

$$
\left(\mathbf{b}^{n}\right)_{i}=\frac{k}{2}\left[\left(g^{n+1}+g^{n}, \chi_{i}\right)+\left(f^{n+1}+f^{n}, v_{i}\right)\right]+\int_{\Omega_{1}} v_{i} U_{h}^{n} d x-\frac{k}{2} a\left(U_{h}^{n}, \phi_{h}^{n} ; v_{i}, \chi_{i}\right)
$$

and $(g, \chi):=\int_{\Gamma} g \chi d s$. For the fully discretized scheme we have the following convergence result.

THEOREM 3. (i) Let $v_{0} \in L^{2}\left(0, T ; H^{1 / 2}(\Gamma)\right), \psi_{0} \in L^{2}\left(0, T ; H^{-1 / 2}(\Gamma)\right), f \in$ $L^{2}\left(0, T ; H^{1}\left(\Omega_{1}\right)^{\prime}\right)$ be given. Then there exists for any $h>0, k>0$ a unique solution $\left\{U_{h}^{n}\right\} \subset H_{h}^{1},\left\{\phi_{h}^{n}\right\} \subset H_{h}^{-1 / 2}, n=0, \ldots, J=T / k$ of $(2.1)$.

(ii) Let $(u, \phi)$ solve $(1.12)$ with $u \in C^{1}\left([0, T] ; H^{r}\left(\Omega_{1}\right)\right) \cap C^{3}\left([0, T] ; L^{2}\left(\Omega_{1}\right)\right)$, $\phi \in C^{0}\left([0, T] ; H^{r-3 / 2}(\Gamma)\right) 1 \leq r \leq d+1$, and $u(0, x)=q(x)$. Let $H_{h}^{1}$ consist of continuous, piecewise polynomials of degree $d$ on a regular partition of $\Omega_{1}$ and let $H_{h}^{-1 / 2}$ consist of piecewise polynomials of degree $d-1$ on the corresponding partition of $\Gamma$. Then there holds with $u^{n}(x):=u(n k, x)$

$$
\max _{0 \leq n \leq J}\left\|U_{h}^{n}-u^{n}\right\|_{L^{2}\left(\Omega_{1}\right)}=O\left(h^{r}+k^{2}\right) .
$$

Furthermore, let $u^{n+1 / 2}(x):=u\left(\left(n+\frac{1}{2}\right) k, x\right), \phi^{n+1 / 2}(x):=\phi\left(\left(n+\frac{1}{2}\right) k, x\right)$ and

$$
U_{h}^{n+1 / 2}:=\frac{1}{2}\left(U_{h}^{n}+U_{h}^{n+1}\right), \quad \phi_{h}^{n+1 / 2}:=\frac{1}{2}\left(\phi_{h}^{n}+\phi_{h}^{n+1}\right)
$$

then we have

$$
\left\{\sum_{n=0}^{J} k\left\|U_{h}^{n+1 / 2}-u^{n+1 / 2}\right\|_{H^{1}\left(\Omega_{1}\right)}\right\}^{1 / 2}=O\left(h^{r-1}+k^{2}\right)
$$

and

$$
\left\{\sum_{n=0}^{J} k\left\|\phi_{h}^{n+1 / 2}-\phi^{n+1 / 2}\right\|_{H^{-1 / 2}(\Gamma)}^{2}\right\}^{1 / 2}=O\left(h^{r-1}+k^{2}\right) .
$$

Proof. The system (2.2) is uniquely solvable since $\mathscr{M}+\frac{k}{2}(A+B)$ is a positive definite matrix. This yields assertion (i). For $n=0,1, \ldots, J$ define $\omega_{1} \in H_{T}^{h}$, $\omega_{2} \in B_{T}^{h}$ by the elliptic projection

$$
a\left(\omega_{1}(t), \omega_{2}(t) ; w, \psi\right)=a\left(u(t), \phi(t) ; w_{h}, \psi_{h}\right) \quad \text { for all } w_{h} \in H_{h}^{1}, \psi_{h} \in H_{h}^{-1 / 2}
$$

and set

$$
\omega_{1}^{n}(x):=\omega_{1}(x, n k), \quad \omega_{2}^{n}(x):=\omega_{2}(x, n k) .
$$


Define $\zeta^{n}:=U_{h}^{n}-\omega_{1}^{n}, \eta^{n}:=\omega_{1}^{n}-u^{n}$ then

$$
e^{n}:=U_{h}^{n}-u^{n}=\zeta^{n}+\eta^{n} \text { and }\left\|\eta^{n}\right\|_{L^{2}\left(\Omega_{1}\right)}=O\left(h^{r}\right) .
$$

Consider the equation $\zeta^{n}$ satisfies where $w_{h} \in H_{h}^{1}, \psi_{h} \in H_{h}^{-1 / 2}$ :

$$
\begin{gathered}
\left(\frac{\zeta^{n+1}-\zeta^{n}}{k}, w_{h}\right)+a\left(\frac{\zeta^{n+1}+\zeta^{n}}{2}, \frac{\phi_{h}^{n+1}-\omega_{2}^{n+1}+\phi_{h}^{n}-\omega_{2}^{n}}{2} ; w_{h}, \psi_{h}\right) \\
=\left(\frac{U_{h}^{n+1}-U_{h}^{n}}{k}, w_{h}\right)+a\left(\frac{U_{h}^{n+1}+U_{h}^{n}}{2}, \frac{\phi_{h}^{n+1}+\phi_{h}^{n}}{2} ; w_{h}, \psi_{h}\right) \\
-\left(\frac{\omega_{1}^{n+1}-\omega_{1}^{n}}{k}, w_{h}\right)-a\left(\frac{\omega_{1}^{n+1}+\omega_{1}^{n}}{2}, \frac{\omega_{2}^{n+1}+\omega_{2}^{n}}{2} ; w_{h}, \psi_{h}\right) .
\end{gathered}
$$

We remark for $w_{h} \in H_{1}^{h}, \psi_{h} \in H_{h}^{-1 / 2}$

$$
a\left(\frac{\omega_{1}^{n+1}+\omega_{1}^{n}}{2}, \frac{\omega_{2}^{n+1}+\omega_{2}^{n}}{2} ; w_{h}, \psi_{h}\right)=a\left(\frac{u^{n+1}+u^{n}}{2}, \frac{\phi^{n+1}+\phi^{n}}{2} ; w_{h}, \psi_{h}\right)
$$

But, for $w_{h} \in H_{1}^{h}, \psi_{h} \in H_{h}^{-1 / 2}$, from (1.12) we obtain

$$
\begin{aligned}
& \left(\frac{U_{h}^{n+1}-U_{h}^{n}}{k}, w_{h}\right)+a\left(\frac{U_{h}^{n+1}+U_{h}^{n}}{2}, \frac{\phi_{h}^{n+1}+\phi_{h}^{n}}{2} ; w_{h}, \psi_{h}\right) \\
& \quad=\int_{\Gamma} g^{n+1 / 2} \psi_{h} d s+\int_{\Gamma} f^{n+1 / 2} w_{h} d s \\
& \quad=\frac{1}{2} \int_{\Omega_{1}}\left(\frac{\partial u^{n}}{\partial t}+\frac{\partial u^{n+1}}{\partial t}\right) w_{h} d x+a\left(\frac{u^{n+1}+u^{n}}{2}, \frac{\phi^{n+1}+\phi^{n}}{2} ; w_{h}, \psi_{h}\right) .
\end{aligned}
$$

Thus from (2.8), (2.9) we have

$$
\begin{aligned}
\left(\frac{\zeta^{n+1}-\zeta^{n}}{k}, w_{h}\right)+a\left(\frac{\zeta^{n+1}+\zeta^{n}}{2}, \frac{\phi_{h}^{n+1}+\phi_{h}^{n}-\left(\omega_{2}^{n+1}+\omega_{2}^{n}\right)}{2} ; w_{h}, \psi_{h}\right) \\
=-\left(\frac{\omega_{1}^{n+1}-\omega_{1}^{n}}{k}, w_{h}\right)+\left(\frac{u_{t}^{n+1}+u_{t}^{n}}{2}, w_{h}\right) \\
\quad+\left(\frac{u^{n+1}-u^{n}}{k}, w_{h}\right)-\left(\frac{u^{n+1}-u^{n}}{k}, w_{h}\right) \\
=-\left(\frac{\eta^{n+1}-\eta^{n}}{k}, w_{h}\right)+\left(r^{n}, w_{h}\right)
\end{aligned}
$$

where $r^{n}:=\frac{1}{2}\left(u_{t}^{n+1}+u_{t}^{n}\right)-\frac{1}{k}\left(u^{n+1}-u^{n}\right)$. 
Next, in (2.10) we set $w_{h}=\zeta^{n+1}+\zeta^{n}$ and $\psi^{h}=\omega_{2}^{n+1}+\omega_{2}^{n}-\left(\phi_{h}^{n+1}+\phi_{h}^{n}\right)$ yielding

$$
\begin{aligned}
\frac{1}{k}\left(\zeta^{n+1}\right. & \left.-\zeta^{n}, \zeta^{n+1}+\zeta^{n}\right)+\frac{1}{2} a\left(\zeta^{n+1}+\zeta^{n}, \phi_{h}^{n+1}+\phi_{h}^{n}-\left(\omega_{2}^{n+1}+\omega_{2}^{n}\right) ; \zeta^{n+1}+\zeta^{n}, \psi_{h}\right) \\
& =\left(\frac{\eta^{n}-\eta^{n+1}}{k}+r^{n}, \zeta^{n+1}+\zeta^{n}\right) .
\end{aligned}
$$

First we will drop the term $a(\cdot, \cdot)$ which is $\geq 0$ by coercivity. But now

$$
\left(\zeta^{n+1}-\zeta^{n}, \zeta^{n+1}+\zeta^{n}\right)=\left\|\zeta^{n+1}\right\|_{L^{2}\left(\Omega_{1}\right)}^{2}-\left\|\zeta^{n}\right\|_{L^{2}\left(\Omega_{1}\right)}^{2}
$$

Also, we use Cauchy-Schwarz inequality and triangle inequality on the right hand side of (2.11) to obtain:

$$
\begin{aligned}
\frac{1}{k}\left\{\left\|\zeta^{n+1}\right\|_{L^{2}\left(\Omega_{1}\right)}^{2}-\left\|\zeta^{n}\right\|_{L^{2}\left(\Omega_{1}\right)}^{2}\right\} \leq & \left(\left\|\frac{\eta^{n+1}-\eta^{n}}{k}\right\|_{L^{2}\left(\Omega_{1}\right)}+\left\|r^{n}\right\|_{L^{2}\left(\Omega_{1}\right)}\right) \\
& \times\left(\left\|\zeta^{n+1}\right\|_{L^{2}\left(\Omega_{1}\right)}+\left\|\zeta^{n}\right\|_{L^{2}\left(\Omega_{1}\right)}\right) .
\end{aligned}
$$

Hence

$$
\left\|\zeta^{n+1}\right\|_{L^{2}\left(\Omega_{1}\right)}-\left\|\zeta^{n}\right\|_{L^{2}\left(\Omega_{1}\right)} \leq\left\|\eta^{n+1}-\eta^{n}\right\|_{L^{2}\left(\Omega_{1}\right)}+k\left\|r^{n}\right\|_{L^{2}\left(\Omega_{1}\right)} .
$$

Summing this inequality from zero to $J$ gives

$$
\left\|\zeta^{n}\right\|_{L^{2}\left(\Omega_{1}\right)} \leq\left\|\zeta^{0}\right\|_{L^{2}\left(\Omega_{1}\right)}+\sum_{n=0}^{J-1}\left\|\eta^{n+1}-\eta^{n}\right\|_{L^{2}\left(\Omega_{1}\right)}+k \sum_{n=0}^{J-1}\left\|r^{n}\right\|_{L^{2}\left(\Omega_{1}\right)} .
$$

Now

$$
\left\|\zeta^{0}\right\|_{L^{2}\left(\Omega_{1}\right)}=O\left(h^{r}\right)
$$

Also we note that

$$
\eta^{n+1}-\eta^{n}=\int_{n k}^{(n+1) k} \frac{d \eta}{d t} d t
$$

Hence

$$
\left\|\eta^{n+1}-\eta^{n}\right\|_{L^{2}\left(\Omega_{1}\right)} \leq \int_{n k}^{(n+1) k}\left\|\frac{d \eta}{d t}\right\|_{L^{2}\left(\Omega_{1}\right)} d t
$$

yielding

$$
\sum_{n=0}^{J-1}\left\|\eta^{n+1}-\eta^{n}\right\|_{L^{2}\left(\Omega_{1}\right)} \leq \int_{0}^{T}\left\|\frac{d \eta}{d t}\right\|_{L^{2}\left(\Omega_{1}\right)} d t \leq C h^{r} \int_{0}^{T}\left\|\frac{\partial u}{\partial t}\right\|_{H^{r}\left(\Omega_{1}\right)} d t .
$$

Here we have used that $\eta=\omega_{1}-u$ satisfies

$$
\left\|\frac{\partial}{\partial t}\left(\omega_{1}-u\right)\right\|_{L^{2}\left(\Omega_{1}\right)} \leq h^{r}\left\|\frac{\partial u}{\partial t}\right\|_{H^{r}\left(\Omega_{1}\right)} .
$$

Hence

$$
\sum_{n=0}^{J-1}\left\|\eta^{n+1}-\eta^{n}\right\|_{L^{2}\left(\Omega_{1}\right)} \leq C h^{r}\left\|\frac{\partial u}{\partial t}\right\|_{L^{\prime}\left(0, T: H^{\prime}\left(\Omega_{1}\right)\right)}=O\left(h^{r}\right) .
$$


Also by Taylor's series we have

$$
\left|\frac{1}{2}\left(\frac{\partial u^{n}}{\partial t}+\frac{\partial u^{n+1}}{\partial t}\right)-\frac{u^{n+1}-u^{n}}{k}\right| \leq C k^{2} \max _{m k \leq t \leq(n+1) k}\left|u_{t t t}\right| .
$$

From (2.15) we obtain

$$
\left\|r^{n}\right\|_{L^{2}\left(\Omega_{1}\right)} \leq C k^{2} \max _{x, t}\left|u_{t t t}\right|
$$

or

$$
k \sum_{n=0}^{J-1}\left\|r^{n}\right\|_{L^{2}\left(\Omega_{1}\right)} \leq C(T) k^{2} \max \left|u_{t t t}\right| .
$$

Combining (2.14) and (2.16) and (2.13) gives with (2.12) the desired result

$$
\left\|\zeta^{n}\right\|_{L^{2}\left(\Omega_{1}\right)}=O\left(k^{2}+h^{r}\right)
$$

Hence

$$
\left\|U_{h}^{n}-u^{n}\right\|_{L^{2}\left(\Omega_{1}\right)} \leq\left\|\zeta^{n}\right\|_{L^{2}\left(\Omega_{1}\right)}+\left\|\eta^{n}\right\|_{L^{2}\left(\Omega_{1}\right)}=O\left(k^{2}+h^{r}\right)
$$

yielding (2.4).

In order to derive the estimates $(2.5),(2.6)$ we proceed as follows. If we introduce $e_{1}^{n}:=\zeta^{n}=U_{h}^{n}-\omega_{1}^{n}, \quad e_{2}^{n}:=\phi_{h}^{n}-\omega_{2}^{n}, \quad \varepsilon_{1}^{n}:=\omega_{1}^{n}-u^{n}, \quad \varepsilon_{2}^{n}:=\omega_{2}^{n}-\phi^{n}$ and make use of the coerciveness of $a(\cdot, \cdot)$ in $(2.11)$ by taking

$$
w_{h}=\zeta^{n+1}+\zeta^{n}=e_{1}^{n}+e_{1}^{n+1}, \quad \psi_{h}=\omega_{2}^{n+1}+\omega_{2}^{n}-\left(\phi_{h}^{n+1}+\phi_{h}^{n}\right)=-e_{2}^{n}-e_{2}^{n+1}
$$

we obtain

$$
\begin{gathered}
\frac{1}{k}\left(\left\|e_{1}^{n+1}\right\|_{L^{2}\left(\Omega_{1}\right)}^{2}-\left\|e_{1}^{n}\right\|_{L^{2}\left(\Omega_{1}\right)}^{2}\right)+\gamma\left(\left\|\frac{e_{1}^{n}+e_{1}^{n+1}}{2}\right\|_{H^{1}\left(\Omega_{1}\right)}^{2}+\left\|\frac{e_{2}^{n}+e_{2}^{n+1}}{2}\right\|_{H^{-1 / 2}(\Gamma)}^{2}\right) \\
\leq C\left(\left\|\frac{\varepsilon_{1}^{n+1}-\varepsilon_{1}^{n}}{k}\right\|_{L^{2}\left(\Omega_{1}\right)}+\left\|r^{n}\right\|_{L^{2}\left(\Omega_{1}\right)}\right)\left(\left\|e_{1}^{n+1}\right\|_{L^{2}\left(\Omega_{1}\right)}+\left\|e_{1}^{n}\right\|_{L^{2}\left(\Omega_{1}\right)}\right) .
\end{gathered}
$$

Now we drop the term in the first bracket on the left hand side of $(2.17)$, then multiply by $k$ and sum over $n$ from 0 to $J-1$. We obtain using the above arguments

$$
\left(\sum_{n=0}^{J-1} k\left\|\frac{e_{1}^{n}+e_{1}^{n+1}}{2}\right\|_{H^{1}\left(\Omega_{1}\right)}^{2}\right)^{1 / 2}+\left(\sum_{n=0}^{J-1} k\left\|\frac{e_{2}^{n+1}+e_{2}^{n}}{2}\right\|_{H^{-1 / 2}(\Gamma)}^{2}\right)^{1 / 2} \leq C\left(k^{2}+h^{r-1}\right) .
$$

With the notation

and

$$
e_{1}^{n+1 / 2}:=\frac{e_{1}^{n+1}+e_{1}^{n}}{2}=U_{h}^{n+1 / 2}-\omega_{1}^{n+1 / 2}
$$

this leads with

$$
e_{2}^{n+1 / 2}:=\frac{e_{2}^{n+1}+e_{2}^{n}}{2}=\phi_{h}^{n+1 / 2}-\omega_{2}^{n+1 / 2}
$$

$$
\left\|U_{h}^{n+1 / 2}-u^{n+1 / 2}\right\|_{H^{1}\left(\Omega_{1}\right)} \leq\left\|e_{1}^{n+1 / 2}\right\|_{H^{1}\left(\Omega_{1}\right)}+\left\|\varepsilon_{1}^{n+1 / 2}\right\|_{H^{1}\left(\Omega_{1}\right)}
$$

to the estimate $(2.5)$. The estimate $(2.6)$ follows similarly. 
Below we present experimental convergence rates for the solution of the discrete system (2.1) using continuous piecewise linear finite element approximations $U_{h}^{n}$ for $u_{1}$ in $\Omega_{1}$ and piecewise constant boundary element approximations $\phi_{h}^{n}$ for $\partial u_{1} / \partial n$ on $\Gamma$. We consider the square $\Omega_{1}=\left\{\left(x_{1}, x_{2}\right) \in \mathbb{R}^{2}:\left|x_{i}\right|<\frac{1}{4}, i=1,2\right\}$ and take the time interval $t \in[0,12]$. The Crank-Nicolson solutions $\left(U_{h}^{n}, \phi_{h}^{n}\right)$ are computed at each time step $k$ on a fixed criss-cross grid on $\Omega_{1}$ and on the induced boundary element mesh on $\Gamma$, respectively.

Example 1. (See Table 1.) $u_{1}=e^{-t / 10}\left(2 t+x_{1}^{2}+x_{2}\right)$ in $\Omega_{1} \times[0,12], u_{2}=$ $e^{-t / 10} \frac{t}{2} \ln \left(x_{1}^{2}+x_{2}^{2}\right)$ in $\left(\mathbb{R}^{2} \backslash \bar{\Omega}_{1}\right) \times[0,12]$. Here we take $k=2 h, T=12$ and $J=\frac{6}{h}$ and define (compare (2.4)-(2.6))

$$
\begin{gathered}
e_{1}:=\max _{0 \leq n \leq J}\left\|U_{h}^{n}-u^{n}\right\|_{L^{2}\left(\Omega_{1}\right)}, \\
e_{2}:=\left\{\sum_{n=0}^{J} k\left\|U_{h}^{n+1 / 2}-u^{n+1 / 2}\right\|_{L^{2}\left(\Omega_{1}\right)}^{2}\right\}^{1 / 2}, \\
e_{3}:=\left\{\sum_{n=0}^{J} k\left\|\phi_{h}^{n+1 / 2}-\phi^{n+1 / 2}\right\|_{L^{2}(\Gamma)}^{2}\right\}^{1 / 2} .
\end{gathered}
$$

Example 2. (See Table 2.)

$$
\begin{gathered}
u_{1}=e^{-t / 10} \frac{1}{t} \exp \left[-\frac{\left(x_{1}-1\right)^{2}+\left(x_{2}-1\right)^{2}}{4 t}\right] \quad \text { in } \Omega_{1} \times[0,12] \\
u_{2}=e^{-t / 10} \frac{t}{2} \ln \left(x_{1}^{2}+x_{2}^{2}\right) \text { in }\left(\mathbb{R}^{2} \backslash \bar{\Omega}_{1}\right) \times[0,12] .
\end{gathered}
$$

\begin{tabular}{|c|c|c|c|c|c|c|}
\hline$h$ & $e_{1}$ & $r_{1}$ & $e_{2}$ & $a_{2}$ & $e_{3}$ & $\alpha_{3}$ \\
\hline $1 / 6$ & .052231 & -2.07 & .148888 & 2.07 & 1.70288 & 4.11 \\
\hline $1 / 8$ & .028816 & 1.99 & .082168 & 1.99 & .522742 & 1.70 \\
\hline $1 / 10$ & .018478 & 2.00 & .052689 & 2.00 & .357865 & 2.59 \\
\hline $1 / 12$ & .012835 & 2.00 & .036599 & 2.00 & .223067 & 2.37 \\
\hline $1 / 14$ & $\begin{array}{l}.009432 \\
.007223\end{array}$ & 2.00 & $\begin{array}{l}.026896 \\
.020599\end{array}$ & 2.00 & .154757 & 2.15 \\
\hline
\end{tabular}

Here we take $k=\frac{4}{5} h, T=12$ and $J=\frac{15}{h}$ and define $e_{1}, e_{2}, e_{3}$ as in (2.18)-(2.20).

TABLE 1. Experimental convergence rates $\alpha_{1}, a_{2}, \alpha_{3}$ for $e_{1}, e_{2}, e_{3}$. 
TABLE 2. Experimental convergence rates $\alpha_{1}, \alpha_{2}, \alpha_{3}$ for $e_{1}, e_{2}, e_{3}$.

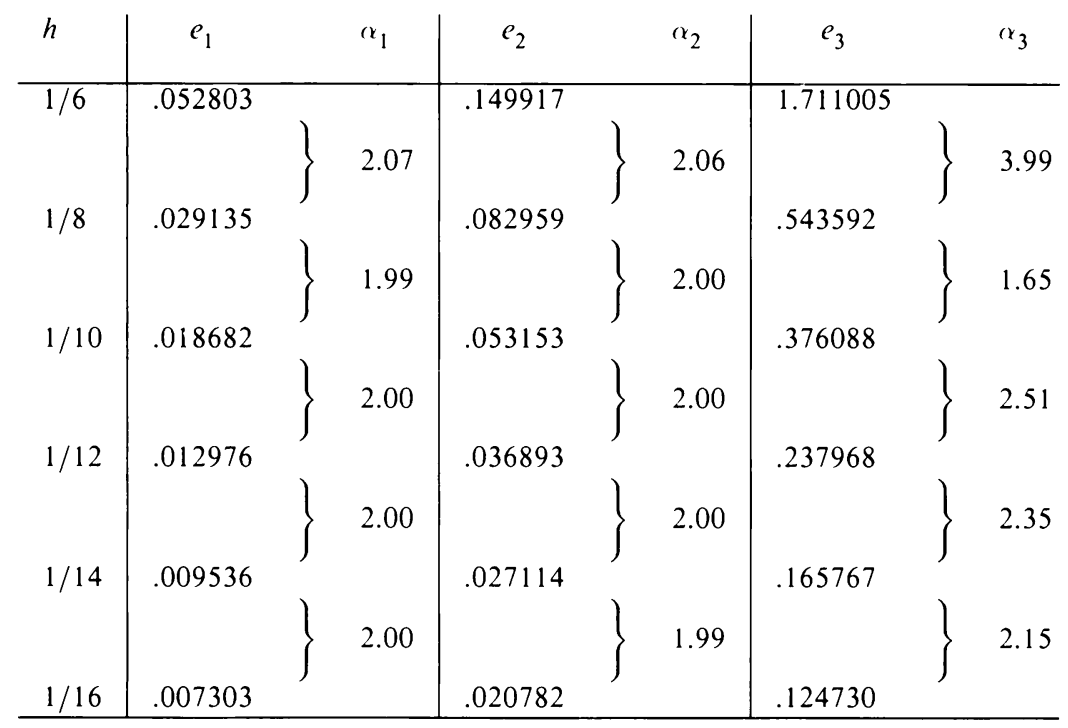

The above computations were performed on the CYBER 855 at Georgia Tech.

Acknowledgments. The first author was partly supported by the DFG Forschergruppe K0634/32-1. The second author was partly supported by the NSF Grant DMS-8704463. The third author was partly supported by the NSF Grants DMS8603954 and DMS-8704463.

\section{REFERENCES}

[1] I. Babuška and A. K. Aziz Survey lectures on the mathematical foundations of the finite element method, The Mathematical Foundations of the Finite Element with Applications to Partial Differential Equations (ed. by A. K. Aziz), Academic Press, New York, 1972, pp.3-259

[2] M. Costabel $A$ symmetric method for the coupling of finite elements and boundary elements, The Mathematics of Finite Elements and Applications IV, MAFELAP 1987 (ed. J. R. Whiteman), Academic Press, London, 1988, pp. 281-288

[3] M. Costabel, On the coupling of finite and boundary element methods, Proc. Intern. Symp. on Numerical Analysis 1987 (eds. O. Celebi, B. Karasözen), METU, Ankara, 1989, pp. 1-14

[4] M. Costabel and E. P. Stephan, Coupling of finite elements and boundary elements for transmission problems of elastic waves in $\mathbb{R}^{3}$, IUTAM Symp. Advanced BEM (eds. T. Cruse, et al.), San Antonio, 1987, Springer Verlag, Berlin, 1987, pp. 117-124

[5] M. Costabel and E. P. Stephan, Coupling of finite elements and boundary elements for inhomogeneous transmission problems in $\mathbb{R}^{3}$. The Mathematics of Finite Elements and Applications IV, MAFELAP, 1987 (ed. J. R. Whiteman), Academic Press, London, 1988, pp. 289-296

[6] H. Han, A new class of variational formulations for the coupling of finite and boundary element methods, Preprint, Univ. Maryland, College Park, 1988

[7] J. Johnson and J. C. Nedelec, On the coupling of boundary integral and finite element methods, Math. Comp. 35, 1063-1079 (1980)

[8] J. L. Lions and E. Magenes, Non-homogeneous Boundary V'alue Problems and Applications I. Springer Verlag. Berlin, 1972

[9] R. C. MacCamy and M. Suri. A time-dependent interface problem for two-dimensional eddy currents, Quart. Appl. Math 44 pp 675-690, 1987 